\section{Do weak stationary magnetic fields affect the perceived astringency of red wine?}

\author{
Stephen Wesley Rowcliffe \\ Faculty of Science, Department of \\ Biology, The United World College of \\ Southeast Asia, Singapore
}

\begin{abstract}
To investigate claims that products containing weak stationary magnets can reduce the astringency of tannic red wine, a double-blind randomized trial was carried out, in which 96 paired tastings were conducted of magnetized and non-magnetized samples of a young Nebbiolo. The data showed no association between reported differences in astringency and actual difference in the magnetic treatments given to the wine $\left(\chi^{2}=0.135\right.$, degrees of freedom $\left.=1, \mathrm{P}=0.71\right)$. This study confirms an earlier work that magnets have no observable effect on red wine.
\end{abstract}

\section{Introduction}

There is a range of commercially available wine magnets which make various claims to enhance the palatability of red wine. Having reviewed the experimental research of Rubin et al. ${ }^{1}$ into the efficacy of magnets on improving cheap wines, a replication study was designed with a more specific focus on the effects of weak magnetic fields on the astringent properties of highly tannic red wine. In their experiment, Rubin and his team used The Perfect Sommelier ${ }^{\mathrm{TM}}$, which consists of a magnetic coaster and stopper. In this experiment, the Wine Clip ${ }^{\mathrm{TM}}$ was used, which consists of a collar made up of six bar magnets placed around the neck of the bottle, through which the wine is poured.

\section{The magnetic properties of the Wine Clip ${ }^{\mathrm{TM}}$}

A Magnaprobe was used to determine the direction of the field lines, which were found to form tightly packed longitudinal lines along the central axis of the device. The lines emerged from the ends of the clip and formed a bar magnet-like arrangement around the exterior, as shown in Figure 1. The maximum field strength measured within the clip was determined using a Vernier Magnetic Field Sensor set to Low amplification (x10). Within the clip along the longitudinal axis, along which the wine would flow, a maximum range of 13.8 $\mathrm{mT} \pm 0.1 \mathrm{mT}$ was recorded.

\section{The claims of the manufacturer}

The manufacturer claims that the Wine Clip $^{\mathrm{TM}}$ results in: i) A smoother, more balanced flavour; softer tannins. ii) An effect that is most noticeable on highly tannic red wines. (http://www.thewineclip.com/howit-works/, accessed $15^{\text {th }}$ Jan 2018).

\section{The proposed mechanism of action of the Wine ClipTM}

The manufacturer claims that these effects occur because: i) passing a conductive fluid through a properly designed magnetic field has an effect on the polar molecules in the fluid; ii) the large, polymerized tannins in wine that normally result in a high degree of astringency are affected in a way that results in a less astringent, softer flavor; iii) (it) accelerates aeration, by drawing higher concentrations of oxygen to the wine as it is being poured. In contrast with most gases, oxygen is highly magnetically susceptible, and is attracted to a magnetic field.

\section{Evaluating the claims of the mecha- nism of action}

Grissom $^{2}$ states that few areas are as controversial as research into the biological effects of magnetic fields; indeed, the literature is full of contradictory reports, with few successful replication studies coupled with a lack of plausible biophysical mechanisms. ${ }^{3}$ Therefore, there is a strong tendency for scientists to approach such claims with a high level of initial skepticism. However, the aim of this paper is to approach the matter with an open mind, and to evaluate each claim on its own merits according to accepted scientific theory and peer-reviewed research.

Claim 1 states that passing a conductive fluid through a magnetic field has an effect on the polar molecules in the fluid. There has been considerable research into the effects of magnetic devices on flowing water in pipes, in particular on the effects of such fields on calcium and magnesium ions in solution. Zamora et $a l .{ }^{4}$ investigated claims that a static magnetic field can affect the $\mathrm{pH}$, conductivity, hardness and surface tension of drinking water, and found no significant change in any of these variables. This finding is consistent with the work of Amiri and Dadkhah, ${ }^{5}$ who conducted over 200 experiments and could find no effect of magnetic fields on the surface tension of water.

On the other hand, in 2007 Holysz et
Correspondence: Stephen Wesley Rowcliffe, UWCSEA, 1207 Dover Road, Singapore 139654.

Tel.: +65.9752.7871

E-mail: sro@uwcsea.edu.sg

Key words: Astringency, magnet, magnetic field, tannins, red wine.

Conflict of interest: the author declares no potential conflict of interest.

Funding: none.

Received for publication: 2 November 2017.

Revision received: 16 January 2018.

Accepted for publication: 17 January 2018.

This work is licensed under a Creative Commons Attribution NonCommercial 4.0 License (CC BY-NC 4.0).

(C) Copyright S. Wesley Rowcliffe, 2017

Licensee PAGEPress, Italy

Wine Studies 2017: 6:7172

doi:10.4081/ws.2017.7172

$a l .{ }^{6}$ reported that a $15 \mathrm{mT}$ magnetic field did have a measurable effect the conductivity of ionic solutions, and attributed these findings to changes in the hydration shells around the ions brought about by the magnetic field somehow interacting with hydrogen bonds between water molecules and the ions. Szcześ et al. ${ }^{7}$ also claim to have detected an increase in conductivity and evaporation rate when distilled water was passed through a $15 \mathrm{mT}$ stationary magnetic field, hypothesizing that this was due to a strengthening of the hydrogen bond network between the water molecules. Similar conclusions were drawn by Ibrahim $^{8}$ and Cai et al., ${ }^{9}$ albeit with much stronger magnetic fields, postulating that larger clusters of water molecules are formed in magnetic fields as a result of stronger hydrogen bond formation. Toledo et al. ${ }^{10}$ theorized that such clusters of water molecules would in fact be smaller, but more numerous, in water exposed to magnetic fields. Iwasaka and Ueno ${ }^{11}$ also found experimental evidence that magnetic fields can affect hydrogen bonds between water molecules, and hydration shells of solvent molecules, although once again in very strong fields of $14 \mathrm{~T}$.

Water is a diamagnetic substance, and is repelled by magnetic fields. Most explanations for how magnetic fields might affect hydrogen bonding derive from the field of spin chemistry, and indeed there is a body of evidence that magnetic fields can alter the rate, yield, or product distribution of chemical reactions $;{ }^{12}$ indeed, many magnet- 
ic field effects are known on reactions occurring in solids or the interior of proteins. ${ }^{12}$ It is, therefore, not outside of the bounds of plausibility that a weak magnetic field could have some effect on the hydrogen bonds between the myriad molecules in wine, however slight.

Claim 2 states that, when exposed to the magnetic field in the Wine Clip ${ }^{\mathrm{TM}}$ the large, polymerized tannins in wine that normally result in a high degree of astringency are affected in a way that results in a less astringent, 'softer' flavor.

Tannins are a diverse group of phenolic compounds found in grape skins, a subset of which, known as flavanols or proanthocyanadins, are responsible for the astringent organoleptic oral sensation associated with red wine, tea, and others. ${ }^{13}$ These molecules may exist as monomers or polymers of up to 80 units, and produce the sensation of astringency by interacting with and deacti- vating the salivary proteins responsible for lubrication of the buccal cavity. The degree of astringency is positively correlated with the degree of flavanol polymerization, ${ }^{14-16}$ and with a higher degree of salivary protein complex formation. ${ }^{17}$ In summary, larger tannin polymers react with a greater number of salivary protein molecues, resulting in greater perceived astringency.

Therefore, for claim 2 to be true, the magnetic field must either reduce the size of

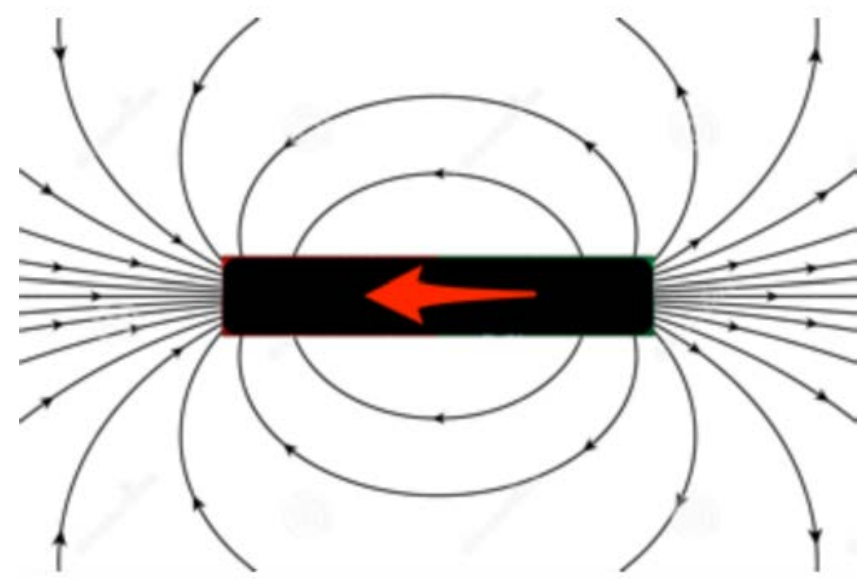

Figure 1. The magnetic field lines around the Wine Clip ${ }^{\mathrm{TM}}$, the direction of flow of wine shown by the red arrow.<smiles>CNC(=O)C1C2CCC(C2)N1C(=O)CNC(C)=O</smiles>

Figure 2. Protein-Tannin interactions. The grey areas show the hydrophobic interactions; the green lines indicate hydrogen bonds. Based on the work of Santos et al. ${ }^{16}$

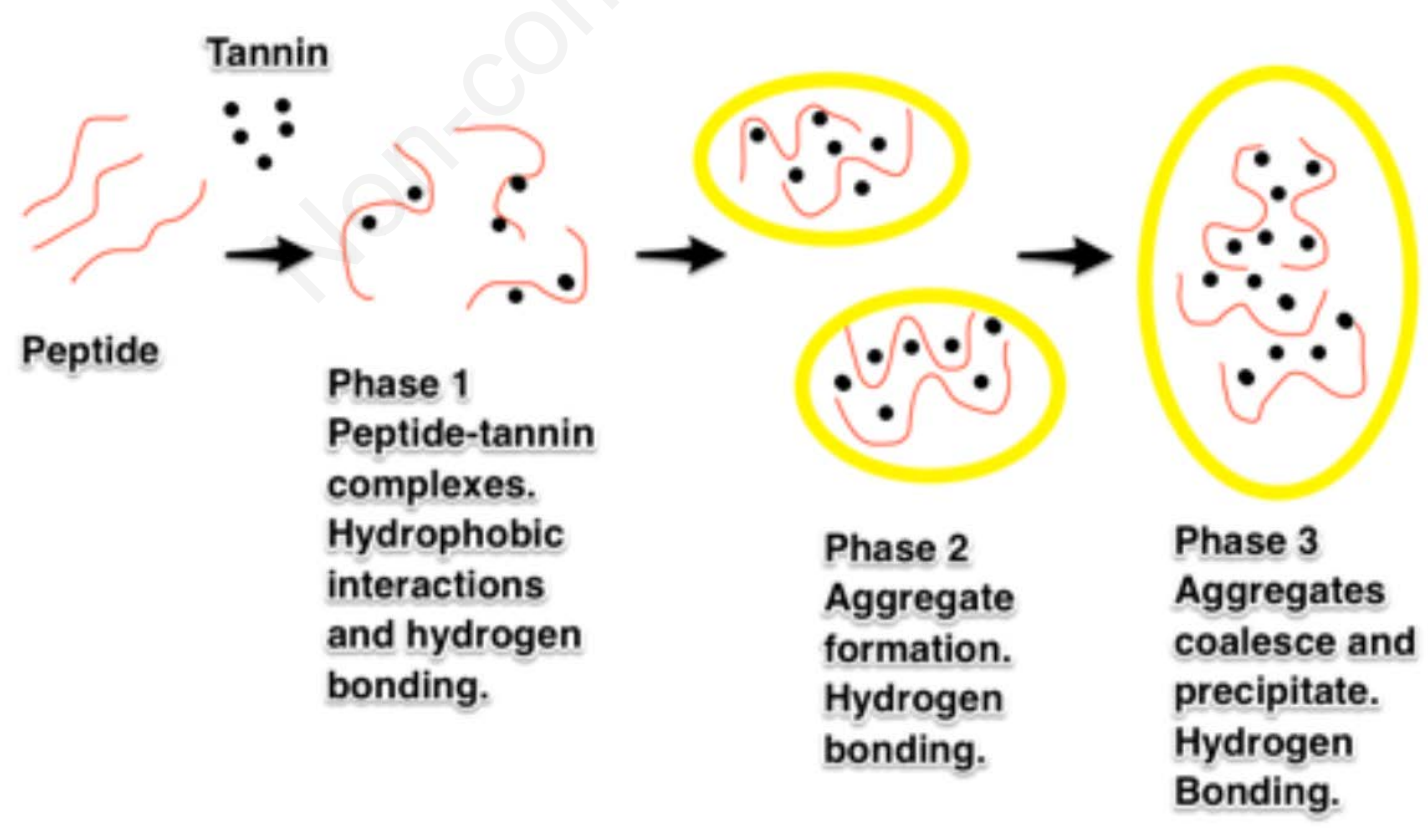

Figure 3. The formation of peptide-tannin complexes as a wine ages. Based on the work of McRae et al. ${ }^{24}$ 
the tannin polymers by increasing the rate of hydrolysis of the bonds between the monomers, or reduce the degree to which tannins interact with salivary proteins. It has been well established that any effect of a static magnetic field on a chemical system would have to be kinetic, rather than thermodynamic, in nature, ${ }^{18}$ and therefore the first possibility may be rejected - it is energetically impossible that a weak magnetic field could break the covalent bonds between flavanol monomers. However, the possibility remains that the magnetic field could reduce the degree to which tannins interact with salivary proteins, and a mechanism by which this might occur is discussed below.

Astringency is defined as a drying and puckering sensation in the mouth and derives from a tactile sensation caused by a loss of lubricity in oral saliva.$^{19}$ Cala et al. ${ }^{20}$ used 3D molecular modeling software to determine that salivary proteins rich in the amino acid proline have three binding sites to which tannins may adhere with high affinity, causing a precipitation of these lubricating proteins from the salivary solution and resultant sensation of roughness in the mouth. Such tannin-protein interactions are thought to be governed by hydrogen bonding between carbonyl groups on the proline residues of salivary proteins and phenol and catechol $\mathrm{OH}$ groups on tannin molecules. ${ }^{21}$ There is also evidence that hydrophobic interactions are important in forming and stabilizing tannin-protein interactions. ${ }^{22}$ The nature of both interactions are shown in Figure 2.

Proline rich proteins are often found in red wine itself, particularly in Cabernet Sauvignon and Italian grape varieties. ${ }^{23}$ McRae et al. ${ }^{24}$ have shown that increased interactions between tannins and prolinerich wine proteins over time may explain the reduction in wine astringency that occurs naturally in a bottle as a wine ages, by the formation and eventual precipitation of protein-tannin complexes in the wine, as shown in Figure 3. Therefore, a hypothesis can be made that, when a wine passes through the Wine Clip ${ }^{\mathrm{TM}}$, the magnetic field strengthens, stabilizes or increases the number of hydrogen bonds between the prolinerich wine proteins and the tannins, giving rise to an increase in either the stability or the number of tannin-protein complexes. This would reduce the number of proteintannin complexes formed between wine tannins and salivary proteins when the wine is tasted, hence reducing the perceived astringency of the wine.

Claim 3 states that the magnetic field draws higher concentrations of oxygen to the wine (because) oxygen is highly magnetically susceptible, and is attracted to a magnetic field. It is true that oxygen is paramagnetic gas, attracted to magnetic fields, and it is also true that oxygenation is known to reduce tannin molecular mass and astringency. ${ }^{25}$ However, Kitazawa et al. ${ }^{26}$ found that significant effects on the solubility of oxygen in water occur only in magnetic fields well above 1T, three orders of magnitude stronger than the $15 \mathrm{mT}$ fields in the Wine Clip ${ }^{\mathrm{TM}}$. It therefore seems highly unlikely that any significant increase in oxygenation of the wine occurs in such a weak field, and so this claim seems highly improbable.

\section{Materials and Methods}

A double blind randomized trial was carried out, in which participants were asked to taste four paired samples of wine and report whether they could detect a difference between the astringency in each of the pairs. As the manufacturer claimed that the effect that is most noticeable on highly tannic red wines, a red wine was selected that is known for its high tannins and astringency, a young Sordo Nebbiolo d'Alba $2014 .{ }^{27}$

\section{Magnetizing the wine}

The first $375 \mathrm{~mL}$ of each bottle of wine was decanted into a standard 1-litre carafe. The magnet was then attached to the neck of the bottle and the remaining $375 \mathrm{~mL}$ of wine was immediately poured into an identical second carafe, to ensure that no magnetized wine would contaminate the non-magnetized wine by backflow. Using half of the same bottle of wine in this way also avoids the possibility that pre-existing differences between bottles of the same wine may mask the effect of the magnet, as suggested by Rubin et al. ${ }^{1}$ in their discussion as a weakness of their own method. A further modification to their method was to add control pairings for false positive and false negative results, where both samples of wine were either magnetized or non-magnetized.

Controlled variables: i) Participants were comparing identical wine - from the same bottle - the only difference being the presence or absence of the magnetic field; ii) The wine pairs were served at the same temperature; iii) Participants compared identical volumes of wine $(20 \mathrm{~mL})$; iv) The wine treatments had been exposed to oxygen for the same duration in the carafes; v)
All participants were university-educated adults with some experience in wine tasting.

\section{Randomizing the trials}

There were four pairing treatments, shown in Table 1.

Rubin et al. ${ }^{1}$ added questions to their survey to determine the wine tasting proficiency of their participants, based on the work of Hughson and Boakes. ${ }^{28}$ These questions were omitted from the design of this experiment, as Rubin and co-workers found that wine-tasting proficiency had no statistically significant effect on their results.

Each participant was given a tray with four pairing treatments, which were allocated using the random number generator function in Microsoft Excel. Table 2 shows the results of the randomization, and Table 3 (for clarity) shows an example of the pairings for Tray 1.

\section{Double blinding}

One experimenter (SR) assembled the trays in the preparation room, behind closed doors from the tasting room. Once a tray was ready, the second experimenter (RH) was summoned into the room to bring the tray to the participant in the tasting room. Thus, neither the participants nor the experimenter who gave them the tray had any knowledge of the treatments. Furthermore, only SR had access to Table 2 or Table 3.

\section{Collecting data}

Participants were given the following instructions and information: i) Astringency is a property of wine that results in a drying and puckering sensation in the mouth; a roughness similar to that which you feel when you drink cold tea; ii) Please taste each pair of glasses of wine in turn and decide whether there is a difference in the astringency you feel between glass A and B, for each of the four rows; iii) Circle yes on the form provided (Table 4) if you feel that there is a difference, and no if the astringency of the two wines feels the same.

Table 1. Pairing treatments and glasses.

\begin{tabular}{lcc} 
Paiving & Glass A & Class B \\
Treatment & & \\
1 & Magnetized & Magnetized \\
2 & Non-magnetized & Non-magnetized \\
\hline 3 & Magnetized & Non-magnetized \\
4 & Non-magnetized & Magnetized \\
\hline
\end{tabular}




\section{Results and Discussion}

These results (Tables 5 and 6) show that the majority of participants reported no discernible difference in the astringency of the wines, whatever the treatment (61/94). Moreover, fewer participants reported a difference in astringency when the wines had received a different magnetic treatment $(16 / 33)$, compared to when the wines had received the same treatment (17/33). Finally, more participants reported detecting no difference in the astringency of the wines when they wines had received different magnetic treatments $(32 / 61)$ than when the treatments had received the same treatment (29/61). These results seem to suggest that the effects of the magnetic treatment on astringency were not detected by the participants, and the results appear to be as a result of random chance alone.

\section{Chi-Squared test}

Chi-square statistic $=0.1354 ; \mathrm{P}$-value $=$ 0.713 - result is not significant; $\mathrm{P}>0.05$.

The Chi-squared test confirms what the data seem to show - that there was no statistically significant association between magnetization treatments and perceived astringency of the wine.

\section{Evaluation and recommendations for further research}

This experiments replicates, builds upon and confirms the findings of earlier research by Rubin et al. ${ }^{1}$ Only one variety of wine was used in this experiment, and further research could investigate whether other wines, for example with lower tannin concentrations or with different blends of tannin molecules, or higher proline-rich protein concentrations, might behave differently in magnetic fields.

The human senses may be a limitation to this experiment. It would be interesting to run samples of magnetized and non-magnetized wine (or indeed purified solutions of tannin molecules and proline-rich proteins) through mass-spectrometry to determine whether the size of tannin-protein aggregations does in fact change as a result of a magnetic field, in a method suggested by the work of Perez-Gregorio et al. ${ }^{29}$ Alternatively, Horne et $a l .{ }^{30}$ describe a method of quantifying interactions between tannins and salivary proteins by the measurement of turbidity, which could also be a viable alternative method for data collection.

\section{Conclusions}

Having reviewed the published literature and suggested a possible causative mechanism by which astringency of red wine could be reduced by magnetic fields, this experiment found no empirical evi-

Table 2. Result of the randomization process of allocating pairings to trays.

\begin{tabular}{lllll} 
Tray & Row 1 & $\begin{array}{c}\text { Pairing Treatment by Row } \\
\text { Row 2 }\end{array}$ & Row 3 \\
1 & 3 & 2 & 1 & 4 \\
2 & 3 & 3 & 3 & 2 \\
\hline 3 & 2 & 4 & 4 & 2 \\
4 & 3 & 4 & 4 & 3 \\
\hline 5 & 3 & 2 & 2 & 4 \\
6 & 2 & 4 & 4 & 4 \\
\hline 7 & 1 & 1 & 4 & 4 \\
8 & 4 & 4 & 2 & 2 \\
\hline 9 & 2 & 4 & 2 & 1 \\
10 & 4 & 2 & 4 & 2 \\
\hline 11 & 1 & 2 & 1 & 2 \\
12 & 2 & 3 & 3 & 1 \\
\hline 13 & 2 & 4 & 3 & 3 \\
14 & 1 & 3 & 1 & 1 \\
\hline 15 & 2 & 2 & 3 & 3 \\
16 & 1 & 3 & 3 & 3 \\
\hline 17 & 3 & 1 & 1 & 3 \\
18 & 4 & 2 & 2 & 4 \\
\hline 19 & 1 & 2 & 4 & 3 \\
20 & 1 & 1 & 2 & 1 \\
\hline 21 & 4 & 2 & 4 & 3 \\
22 & 2 & 3 & 1 & 1 \\
\hline 23 & 3 & 1 & 3 & 3 \\
\hline & & & & \\
\hline
\end{tabular}

Table 3. The pairings for Tray 1.

\begin{tabular}{lcc} 
Row & Glass A & Glass B \\
1 & Magnetized & Non-magnetized \\
2 & Non-magnetized & Non-magnetized \\
\hline 3 & Magnetized & Magnetized \\
4 & Non-magnetized & Magnetized \\
\hline
\end{tabular}

Table 4. Response form.

\begin{tabular}{lcc} 
Did you notice a difference in the \\
astringency between wines $\mathrm{A}$ and $\mathrm{B}$ ? \\
Row 1 & YES & NO \\
Row 2 & YES & NO \\
\hline Row 3 & YES & NO \\
Row 4 & YES & NO \\
\hline
\end{tabular}

4

dence that human subjects can detect such an effect, if it exists. It seems reasonable to conclude, therefore, that either tannin molecules in red wine are not affected at all by such magnetic fields, or that any effects on the astringency of a tannic young Nebbiolo are too subtle to be detected by the human
Table 5. Raw data; total number of responses by pairing treatment.

\begin{tabular}{lcc} 
Treatment & $\begin{array}{l}\text { Number of Responses } \\
\text { Yes }\end{array}$ & No \\
1 & 8 & 13 \\
2 & 9 & 16 \\
\hline 3 & 8 & 15 \\
\hline 4 & 8 & 17 \\
\hline
\end{tabular}

Table 6. Processed data; total number of responses, pairing treatments grouped according to whether the wines were both magnetised or non-magnetised (Same) or one magnetized and the other non-magnetized (Different).

\begin{tabular}{lccc} 
& \multicolumn{3}{c}{ Number of Responses } \\
Treatment & Yes & No & Total \\
Same (1/2) & 17 & 29 & 46 \\
Different (3/4) & 16 & 32 & 48 \\
\hline Total & 33 & 61 & 94 \\
\hline
\end{tabular}


palate. In either case, this study provides more evidence that the extravagant claims made by wine magnet manufacturers should be treated with the utmost skepticism by the consumer. The reported efficacy of the device in consumer reviews is most likely a result of the placebo effect resulting from the taster seeing the wine magnet on the bottle; it has been found that, for sighted judgment, $95 \%$ of a wine's quality rating may be attributed to the extrinsic visual cues of price and region. ${ }^{31}$

\section{References}

1. Rubin G, Hahn G, Allberry E, Wessely S. Drawn to drink: A double-blind randomised cross-over trial of the effects of magnets on the taste of cheap red wine. J Wine Res 2005;16:65-70.

2. Grissom CB. Magnetic field effects in biology: a survey of possible mechanisms with emphasis on radical-pair recombination. Chem Rev 1995;95:324.

3. Harris SR, Henbest KB, Maeda K, et al. Effect of magnetic fields on cryptochrome-dependent responses in Arabidopsis thaliana. J R Soc Interface 2009;6:1193-205.

4. Zamora LL, Fos GA, López PA, Algarra RM. Magnetized water: Science or fraud? J Chem Educ 2008;85:1416-8.

5. Amiri MC, Dadkhah AA. On reduction in the surface tension of water due to magnetic treatment. Colloids Surfaces A: Physicochem Engin Aspects 2006;278:252-5.

6. Holysz L, Szczes A, Chibowski E. Effects of a static magnetic field on water and electrolyte solutions. J Colloid Interface Sci 2007;316:9961002.

7. Szcześ A, Chibowski E, Hołysz L, Rafalski P. Effects of static magnetic field on water at kinetic condition. Chem Engin Process: Proc Intens 2011;50:124-7.

8. Ibrahim IH. Biophysical properties of magnetized distilled water. Egypt J Sol
2006;29:363-9.

9. Cai R, Yang H, He J, Zhu W. The effects of magnetic fields on water molecular hydrogen bonds. J Mol Struct 2009; 938:15-9.

10. Toledo EJ, Ramalho TC, Magriotis ZM. Influence of magnetic field on physicalchemical properties of the liquid water: insights from experimental and theoretical models. J Mol Struct 2008;888: 409-15.

11. Iwasaka M, Ueno S. Structure of water molecules under $14 \mathrm{~T}$ magnetic field. J Appl Phys 1998;83:6459-61.

12. Rodgers CT. Magnetic field effects in chemical systems. Pure Appl Chem 2009;81:19-43.

13. Cheynier V, Dueñas-Paton M, Salas E, et al. Structure and properties of wine pigments and tannins. Am J Enol Viticult 2006;57:298-305.

14. Noble A. Why do wines taste bitter and feel astringent? Chem Wine Flavor 1998;12:156-65.

15. Vidal S, Francis L, Guyot S, et al. The mouth-feel properties of grape and apple proanthocyanidins in a wine-like medium. J Sci Food Agric 2003;83:56473.

16. Santos-Buelga C, De Freitas V. Influence of phenolics on wine organoleptic properties. In: MorenoArribas MV, Polo MC, eds. New York, NY: Springer, 2009. pp. 529-570.

17. Sarni-Manchado P, Cheynier V, Moutounet M. Interactions of grape seed tannins with salivary proteins. J Agric Food Chem 1999;47:42-7.

18. Atkins PW, Lambert TP. The effect of a magnetic field on chemical reactions. Annual Reports on the Progress of Chemistry, Section A: Physical and Inorganic Chemistry 1975;72:67-88.

19. McRae JM, Kennedy JA. Wine and grape tannin interactions with salivary proteins and their impact on astringency: a review of current research. Molecules 2011;16:2348-64.

20. Cala O, Fabre S, Pinaud N, et al. Towards a molecular interpretation of astringency: synthesis, 3D structure, colloidal state, and human saliva pro- tein recognition of procyanidins. Planta Med 2011;77:1116-22.

21. Simon C, Barathieu K, Laguerre M, et al. Three-dimensional structure and dynamics of wine tannin- saliva protein complexes. A multitechnique approach. Biochemistry 2003;42:10385-95.

22. Oh HI, Hoff JE, Armstrong GS, Haff LA. Hydrophobic interaction in tanninprotein complexes. J Agric Food Chem 1980;28:394-8.

23. Ough, CS. Proline content of grapes and wines. Vitis 1968;7:321-331.

24. McRae JM, Falconer RJ, Kennedy JA. Thermodynamics of grape and wine tannin interaction with polyproline: implications for red wine astringency. J Agric Food Chem 2010;58:12510-8.

25. McRae JM, Day MP, Bindon KA, et al. Effect of early oxygen exposure on red wine colour and tannins. Tetrahedron 2015;71:3131-7.

26. Kitazawa K, Ikezoe Y, Uetake H, Hirota N. Magnetic field effects on water, air and powders. Phys B: Condensed Matter 2001;294:709-14.

27. Asproudi A, Piano F, Anselmi G, et al. Proanthocyanidin composition and evolution during grape ripening as affected by variety: Nebbiolo and Barbera cv. OENO One 2015;49:59-69.

28. Hughson AL, Boakes RA. Perceptual and cognitive aspects of wine expertise. Austral J Psychol 2001;53:103-8.

29. Perez-Gregorio MR, Mateus N, De Freitas V. Rapid screening and identification of new soluble tannin-salivary protein aggregates in saliva by mass spectrometry (MALDI-TOF-TOF and FIA-ESI-MS). Langmuir 2014;30: 8528-37.

30. Horne J, Hayes J, Lawless HT. Turbidity as a measure of salivary protein reactions with astringent substances. Chem Senses 2002;27:653-9.

31. Priilaid DA. Wine's placebo effect: How the extrinsic cues of visual assessments mask the intrinsic quality of South African red wine. Int $\mathrm{J}$ Wine Marketing 2006;18:17-32. 